\title{
It's just my opinion
}

In my last leader I looked at the delicate balance all journals must keep between the inappropriate publication of material that could be damaging to the population (like when the Lancet published the paper on the MMR vaccine which has now been discredited) and adopting too cautious an approach resulting in stagnation of progress. I suggested the peer review process was the method by which this balance could be maintained. I am convinced of this now, after 12 years in post, and I believe that all papers should be peer reviewed, even those submitted for a 'personal opinion' section.

This might almost seem to be a contradiction in terms. Surely opinion papers should enable people to express their opinion, regardless of the current establishment view? To suppress ideas simply because they do not fit our current paradigm of thinking might well seem like caution. Yet, as expressed in my last leader, indiscriminate publishing of an opinion paper just because authors think they are right can be negligent on the part of the editor. Peer review seems to be the best independent way forward, as the editor's own opinions may biased. Which brings me to the point of this leader - what exactly is peer review?

In essence peer review is simply sending a manuscript to independent experts in the field to check the validity and accuracy of the manuscript. Most journals who practise peer review will send a manuscript to at least two referees. Many editors of scientific journals are themselves researchers and scientists, enabling them to judge the relevance and accuracy of the reports from the referees and make a judgement on the manuscript. Because the editor of the $B D J$ is not necessarily a scientist (as I am not) we have a scientific editor to check on the referees and to provide guidance for the editor.

The process is not as complex as it sounds. After a manuscript is submitted I will select two independent experts and send a copy of the manuscript to each (these days most of this is electronic over the internet). They check the manuscript and send me their report, sometimes with advice as to whether to accept or reject the manuscript. I send both reports and my own observations to the scientific editor, who provides me with a further report giving me guidance on how to intepret the reports from the referees. I make the final decision and write to the author(s) of the manuscript with my decision. If I am prepared to accept the manuscript (usually subject to modifications suggested by the referees) then I inform the author(s) who make the necessary changes and resubmit the manuscript. Usually I then accept it. If I am not prepared to accept the manuscript then it is rejected.

The main reason for going through this process is that most manuscripts that are accepted will be improved providing a better final result for readers. This is obviously beneficial, although it may not be appreciated by all readers who are unaware that it happens. The second reason is that manuscripts that end up being published will have been carefully checked to ensure that the science is accurate and appropriate and the research was relevant and carried out to a high standard. In other words, the conclusions of the research that are reported in the paper are more likely to be valid.

The process can be quite taxing for authors, who see their precious and much-tended work diappear into the editorial office for what seems like ages, only to reappear with comments from people that can sometimes sound harsh, unfair and occasionally actually wrong. If the paper is then rejected the author(s) will almost certainly be disheartened.

But this is the price for protection of the readers, because a paper or article in a peer reviewed journal has the benefit of scientific credibility behind it. This applies even more to an opinion paper, even though (as I stated earlier) people may imagine that an opinion paper should be a licence to write what you want. Not so. The referees are there to make sure that all papers, opinion, practice and research, are based on sound scientific principles rather than pure speculation. That is why the whole process can appear complex, longwinded and occasionally harsh.

The final result, though, is that the readers can trust the comments even though they may not agree with them. If that is so, peer review is well worth the effort.

Mike Grace, Editor

m.grace@bda.org

doi: 10.1038/sj.bdj.4811316 